Marco Conedera ${ }^{1}$

Nicola BOMIO-PACCIORINI ${ }^{1}$

Pietro BOMIO-PACCIORINI ${ }^{1}$

Stéphane SCIACCA ${ }^{1}$

Lindo GRANDI ${ }^{1}$

Amadou BoureIMA ${ }^{2}$

Anna Maria Vettraino ${ }^{1}$

${ }^{1}$ Associazione Deserto Verde

Burkinabé

6763 Mairengo

Suisse

${ }^{2}$ Reach BF

06 BP 9904

Ouagadougou

Burkina Faso

\title{
Reconstitution des écosystèmes dégradés sahéliens
}

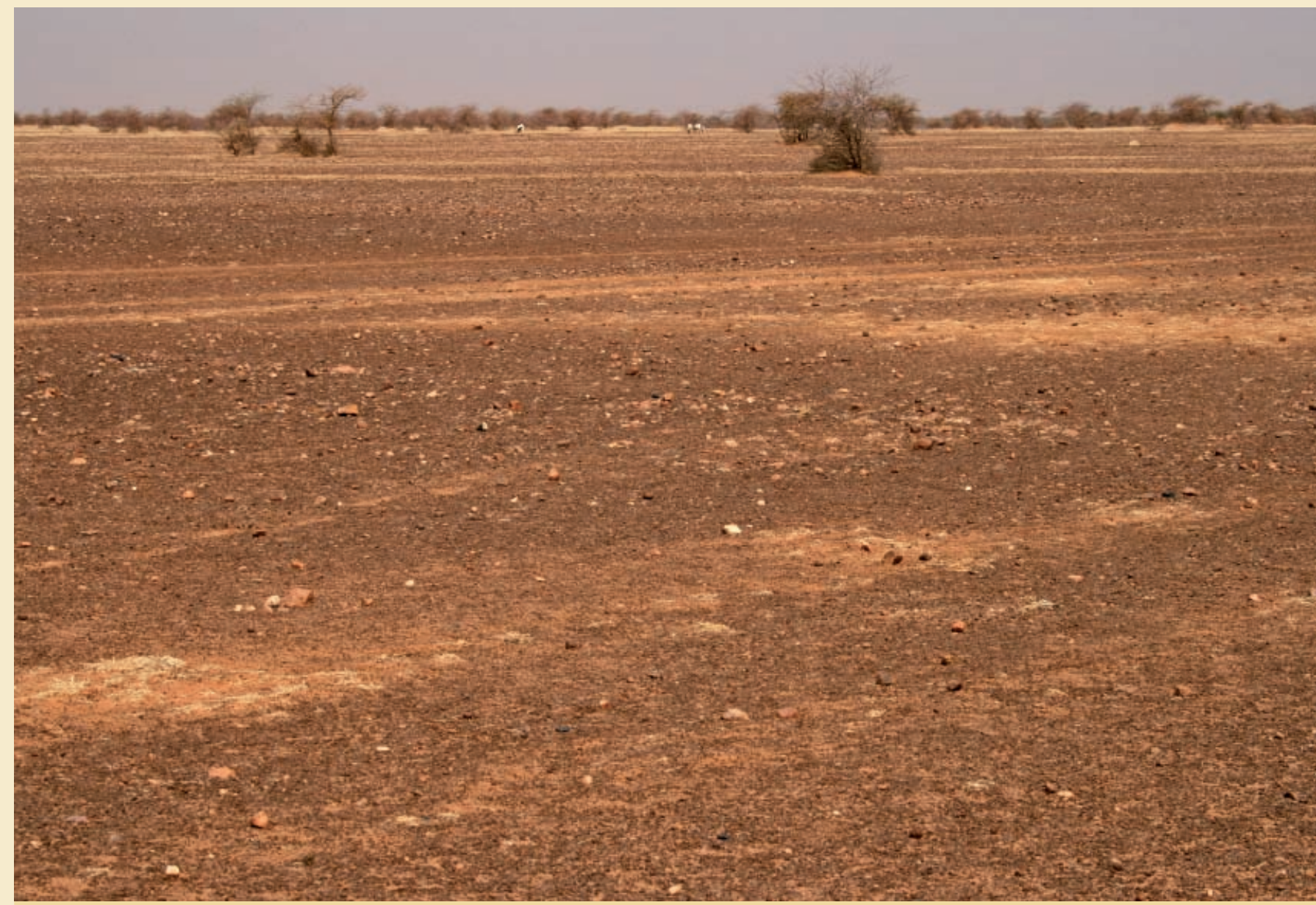

Photo 1.

Surface dégradée sans aucune couverture végétale.

Photo Deserto Verde Burkinabé. 


\section{RÉSUMÉ}

\section{RECONSTITUTION DES ÉCOSYSTÈMES DÉGRADÉS SAHÉLIENS}

La longue période de déficit hydrique dont a souffert la région sahélienne depuis les années 1970 et la sédentarisation progressive des tribus nomades ont localement causé une dégradation et une surexploitation de ces écosystèmes fragiles et notamment de la savane arbustive. Les écosystèmes sont considérés comme dégradés lorsqu'ils perdent leur résilience en subissant des modifications difficilement réversibles ou totalement irréversibles. Les caractéristiques des écosystèmes dégradés sont la raréfaction ou la disparition de la flore arborée et herbacée, la réduction de l'activité biologique du sol et l'augmentation des phénomènes de ruissellement superficiel et d'érosion. La réhabilitation de la couverture végétale des surfaces dégradées représente un passage clé dans le contrôle et la lutte contre la désertification. L'article présente les résultats d'un suivi sur six ans de la récupération d'écosystèmes dégradés dans le nord du Burkina Faso (province de l'Oudalan). Les résultats démontrent l'importance de l'approche basée sur la création de demi-lunes pour la récolte des eaux de pluie dans la restauration des paysages sahéliens et cela même dans les zones les plus dégradées. Sur les surfaces traitées sont constatées une production soutenue de biomasse herbacée (en moyenne environ 1000 à 1200 kg de matière sèche par hectare et par an) et une couverture forestière significative (de l'ordre de 350 arbres par hectare), avec des arbres en mesure de pousser spontanément, même sous la pression du pâturage extensif traditionnel et des années de stress hydrique.

Mots-clés : dégradation du sol, restauration, régénération forestière, désertification, Burkina Faso, Sahel.

\section{ABSTRACT}

\section{RESTORING DEGRADED ECOSYSTEMS IN THE SAHEL}

The prolonged period of water stress experienced by the Sahel since the 1970 s and the gradual sedentarisation of nomadic tribes have caused local degradation and over-exploitation of the region's fragile ecosystems, especially shrub savannah. Ecosystems are assessed as degraded when they lose their resilience as a result of changes that can only be reversed with difficulty, if at all. Degraded ecosystems are characterised by increasing scarcity or complete loss of woody and herbaceous flora, reduced biological soil activity and increased surface run-off and erosion. Restoring plant cover on degraded surfaces is critical to controlling and halting desertification. This article describes the results of a six-year programme to monitor the recovery of degraded ecosystems in northern Burkina Faso (Oudalan province). The results show the value, when restoring even the most degraded Sahelian landscapes, of the approach involving the creation of semicircular depressions to collect rainwater. The areas treated in this way showed sustained production of herbaceous biomass (about 1000 to 1200 kilograms of dry matter per hectare and per year, on average) and significant forest cover (about 350 trees per hectare), with trees capable of spontaneous growth even under pressure from traditional extensive grazing and years of water stress.

Keywords: soil degradation, restoration, forest regeneration, desertification, Burkina Faso, Sahel.

\section{RESUMEN}

\section{RECONSTITUCIÓN DE LOS ECOSISTEMAS DEGRADADOS SAHELIANOS}

El largo período de déficit hídrico padecido por la región saheliana desde los años 70 y la progresiva sedentarización de las tribus nómadas provocaron localmente una degradación y sobreexplotación de estos frágiles ecosistemas y, especialmente, de la sabana arbustiva. Los ecosistemas se consideran degradados cuando pierden su resiliencia al ocasionarse cambios difícilmente reversibles 0 totalmente irreversibles. Los ecosistemas degradados se caracterizan por la rarefacción o desaparición de la flora arbórea y herbácea, la reducción de la actividad biológica del suelo y el aumento de los fenómenos de escorrentía superficial y erosión. La rehabilitación de la cubierta vegetal de las áreas degradadas es un elemento clave para el control y la lucha contra la desertificación. El artículo presenta los resultados de un seguimiento de seis años en la recuperación de ecosistemas degradados en el norte de Burkina Faso (provincia de Oudalan). Los resultados demuestran la importancia del enfoque basado en la creación de medias lunas para la recolección de aguas pluviales en la restauración de los paisajes sahelianos, incluso en las zonas más degradadas. En las áreas tratadas se observó una producción sostenida de biomasa (promedio de aprox. 1000 a 1200 kg de materia seca por hectárea y año) y una cubierta forestal significativa (de unos 350 árboles por hectárea) con árboles capaces de crecer espontáneamente, incluso bajo la presión del pastoreo extensivo tradicional y en años de estrés hídrico.

Palabras clave: degradación del suelo, restauración, regeneración forestal, desertificación, Burkina Faso, Sahel. 


\section{Introduction}

Le Sahel africain (en arabe « rive ») est une zone de transition entre le Sahara aride au nord et la savane (sub)humide au sud. Cette transition se caractérise par un gradient de précipitations qui se traduit par un continuum de changement de formes et d'espèces végétales. La production de biomasse se concentre pendant la saison estivale des pluies. Les contraintes environnementales principales de cette région sont la pénurie, l'irrégularité et l'imprévisibilité des précipitations. Au cours de l'histoire, ces conditions cadres ont forcé les populations autochtones à développer une stratégie sociale et environnementale de survie très flexible, stratégie fondée sur une mobilité extrême et une diversification des revenus, passant du nomadisme à la transhumance, au pastoralisme extensif, à l'agriculture saisonnière du millet (Panicum miliaceum L.) et du sorgho (Sorghum spp.), jusqu'à l'artisanat, le petit commerce et l'émigration saisonnière en périodes sèches. L'économie traditionnelle sahélienne qui en résulte repose principalement sur l'élevage extensif, la production agricole de saison des pluies et la cueillette. Dans ce contexte, les espèces ligneuses constituent une réserve fourragère stratégique pour le bétail pendant la saison sèche, lorsque les herbes des pâturages et les réserves en aliments font défaut (LIKKE et al., 2004).

La variabilité des précipitations et les épisodes de sécheresse sont des caractéristiques normales pour des climats arides et semi-arides. Cependant, la période de sécheresse extrême qui a touché le Sahel depuis la fin des années 1960 ainsi que la séquence d'années de forts déficits pluviométriques exceptionnelles qui se sont succédé avec les sécheresses de 1973, 1984 et 1990 sont à considérer comme tout à fait exceptionnelles (NICHOLSON, 2000). Cette période de sécheresse sans précédent a engendré un déplacement général des isohyètes vers le sud, créant une contrainte supplémentaire pour les délicats écosystèmes sahéliens déjà en équilibre précaire (Goudet, 1985 ; HuLme et al., 2000 ; GONZALEZ, 2001 ; HERRMANn et al., 2005). De fait, la précarité des récoltes a amené certaines populations agricoles à passer à l'agropastoralisme. Cela a augmenté la diversité des ressources mais a surtout exercé une pression accrue sur un écosystème déjà précaire et fragile. La couverture végétale des écosystèmes s'est ainsi réduite en termes de densité de plantes et de diversité des espèces (IEMVT, 1977 ; SANKARAN et al., 2005).

Malgré une augmentation des précipitations au Sahel et un reverdissement de la végétation à partir des années 1990 (oLsson et al., 2005), des analyses de la végétation à long terme ainsi que les témoignages de la population autochtone démontrent de façon indiscutable l'existence à l'échelle locale de poches de dégradation accélérée et continue des terres conduisant parfois à la désertification (HERRMANN et al., 2005 ; HOUNTONDJ et al., 2006 ; ToutAin et al., 2006). Les écosystèmes sont considérés comme dégradés lorsqu'ils perdent partiellement ou totalement de leur résilience, c'est-à-dire de leur capacité à revenir à un état normal de fonctionnement et de structure. Les symptômes typiques de la dégradation des terres sont la raréfaction voire la disparition de la flore herbacée et arborée, la réduction de la taille des arbres qui résistent, la réduction de l'activité biologique du sol et l'augmentation des phénomènes de ruissellement superficiel et d'érosion (TouTAIN et al., 2006).

La restauration de la couverture végétale des surfaces dégradées est un facteur clé de la récupération des écosystèmes dégradés et de la lutte contre la désertification (GouDET, 1985). Dans cet article sont présentés les résultats de six années de suivi de l'efficacité d'un système de labour avec semis d'arbres forestiers. Cette méthode est inspirée de la tradition paysanne des régions sèches qui consiste à concentrer les eaux de pluie dans des dépressions artificielles du sol pour y favoriser la germination et la croissance des espèces herbacées et ligneuses. Cette technique que l'on retrouve notamment dans le « zaï » est une forme particulière de culture en poquets qui concentre l'eau et la fumure dans des micro-bassins où les graines sont semées.

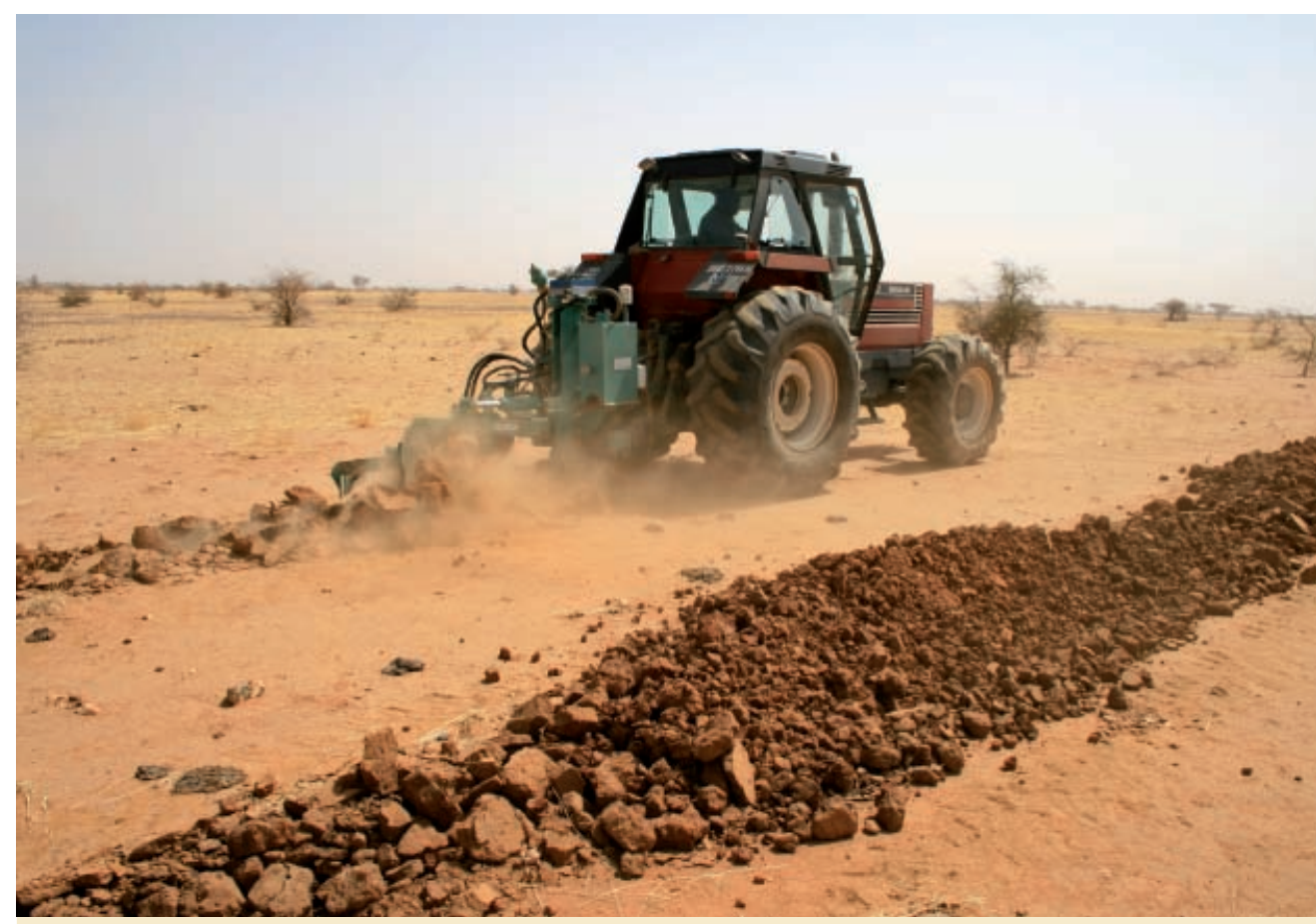

Photo 2

Charrue Delfino en action (champ M, 2008).

Photo Deserto Verde Burkinabé. 


\section{Matériel et méthode}

\section{Site d'étude}

Les activités de restauration du milieu ont été réalisées au nord de Gorom-Gorom (province de l'Oudalan, Burkina Faso) (figure 1) sur des terres dégradées utilisées traditionnellement pour le pâturage et qui, depuis des décennies, présentent une faible densité d'arbres (moins de 50 arbres par hectare) ainsi qu'une couverture herbacée presque totalement absente. Des traces d'érosion éolienne et hydrique importantes se traduisent par une importante présence de cailloux en surface (photo 1).

La province jouit d'un climat qui se situe entre les isohyètes 400 et 600 millimètres (figures 1 et 2) et se caractérise par une température moyenne annuelle de $30^{\circ} \mathrm{C}$. Les précipitations sont concentrées sur la période juillet-août (figure 3), avec un fort gradient négatif vers le nord et une large variation annuelle, saisonnière et surtout spatiale. La distribution des pluies est devenue très irrégulière, en particulier au cours des dernières décennies. Le milieu physique dégradé est dominé par des glacis situés entre 280 et 350 mètres (m) d'altitude sur sols sableux à argilo-sableux, peu profonds et à perméabilité extrêmement limitée en raison notamment de la compaction des horizons de surface (WITTIG et al., 2002). Sur ces glacis, la couverture végétale est représentée par une steppe arbustive à structure ouverte avec une couverture irrégulière d'épineux arborescents dominés par Acacia tortilis (Forssk.) Hayne, Acacia senegal (L.) Willd., Acacia seyal Delile, Ziziphus mauritania Lam., Balanites aegyptiaca (L.) Delile. La population est, pour la majorité, représentée par les Fulanis (Peuls et assimilés) avec une présence significative de Tamacheks (Bella) et de Songhaï. Les Fulanis d'origine nomade, entre-temps sédentarisés, vivent maintenant d'élevage extensif, de la culture du millet et du sorgho, de l'artisanat, du petit commerce et du travail saisonnier dans les régions voisines et à l'étranger (BoLWING et al., 1999 ; HENRY et al., 2004).

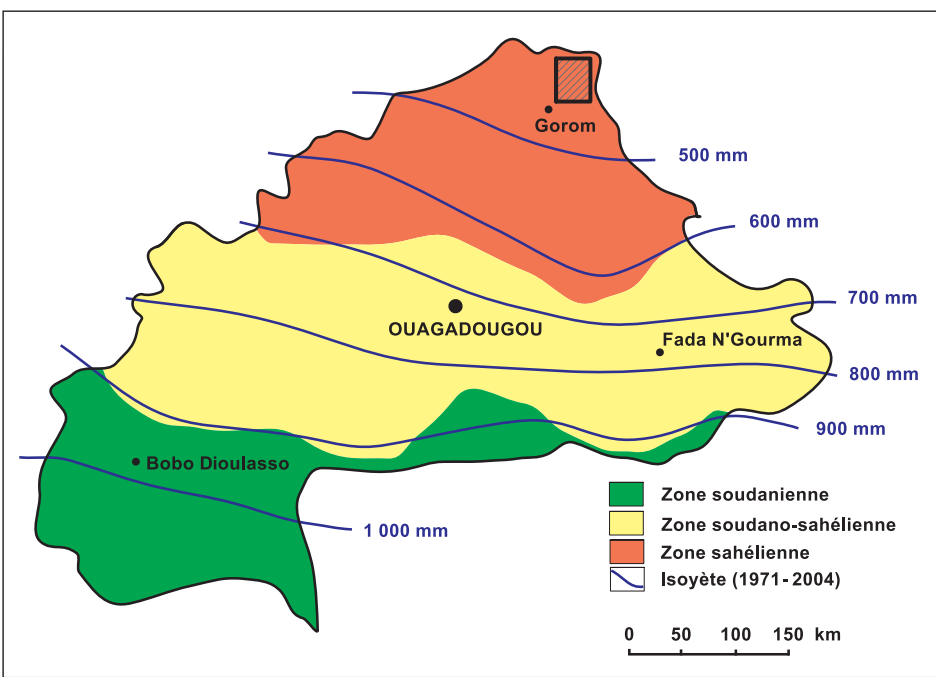

Figure 1.

Carte générale du Burkina avec les isohyètes et la zone d'étude.

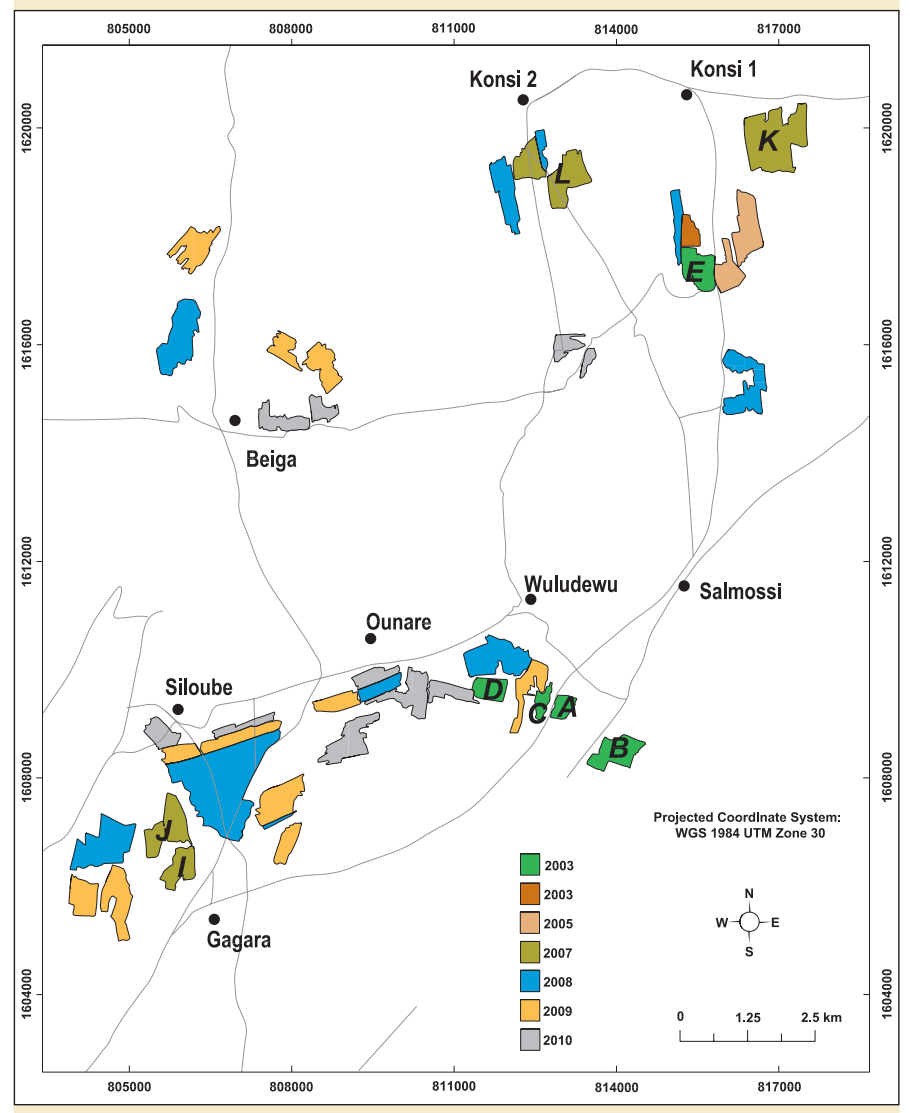

Figure 2.

Carte du site d'étude et des champs labourés par Deserto Verde Burkinabé entre 2003 et 2009. Les lettres indiquent les champs utilisés pour le suivi et les expérimentations.

\section{Le programme de récupération des surfaces dégradées}

L'organisation Reach Burkina Faso a entamé, à partir de l'année 1998, un programme de récupération de surfaces dégradées dans la province de l'Oudalan, avec l'emploi de la charrue Delfino (VALLERANI, 2009) qui, à l'heure actuelle, a permis de récupérer près de 5000 hectares (ha) de terres à des fins agrosylvopastorales. En 2003, l'association suisse Deserto Verde Burkinabé ${ }^{1}$, dont l'objectif principal est la récupération de terres dégradées dans la zone sahélienne du Burkina Faso, a démarré en collaboration avec Reach Burkina Faso son propre programme décennal visant non seulement la réhabilitation environnementale (figure 2), mais également l'évaluation à long terme de l'efficacité de la méthode employée.

L'approche originale consiste à labourer mécaniquement des terres dégradées au moyen de la charrue Delfino (photo 2). Grâce à un mouvement oscillatoire continu à la verticale du coutre, cette charrue est en mesure de creuser des demi-lunes d'un volume d'environ mille litres, aptes à recueillir et maintenir les eaux de pluie et de ruissellement pendant et après la saison des pluies (photo 3). Les labours sont effectués perpendiculairement à la pente (direction du ruissellement) avec un écartement variant de 3 à $6 \mathrm{~m}$ entre chaque ligne de demi-lunes en fonction de la pluviosité, de 


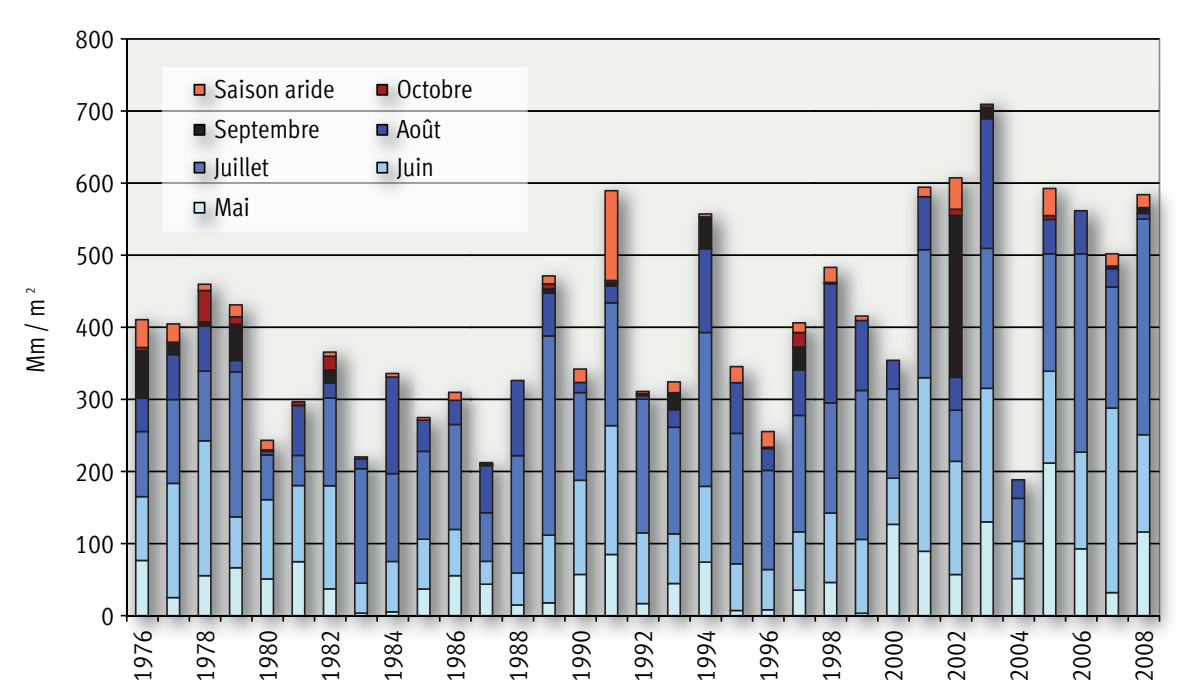

Figure 3.

Précipitations (en millimètres) à Gorom-Gorom (Oudalan) pendant la période 1976-2008 (source : Direction de la météorologie, Ouagadougou).

Ce sont les populations locales qui choisissent elles-mêmes, en fonction de leurs besoins, les surfaces dégradées à récupérer et les espèces ligneuses à introduire, qui récoltent les graines des espèces locales (Acacia tortilis, Balanites aegyptica et Ziziphus mauritania) et les déjections des animaux domestiques et qui exécutent les semis (photo 6). Les populations locales nous indiquent également les espèces ligneuses qu'elles souhaiteraient voir introduites et dont les graines non disponibles sur place devaient être achetées auprès du Cnsf à Ouagadougou.

Le temps machine nécessaire pour ce programme varie de manière importante en fonction notamment des distances à parcourir par le tracteur depuis son dépôt, de sa puissance et de son état d'usure, de l'expérience du tractoriste, du type de sol et du degré de dégradation des

la pente et de la fertilité du sol (photo 4). Les graines des espèces ligneuses sont semées avant la saison des pluies directement dans les demi-lunes creusées par la charrue sans aucun labour ultérieur. Entre les années 2003 et 2006, les semis ont été effectués à l'aide d'une canne semeuse - tube de 180 centimètres $(\mathrm{cm})$ de long - que l'on enfonce de 10 $\mathrm{cm}$ dans la terre et dans laquelle on insère une poignée de 5 à 10 graines d'un mélange de différentes espèces de ligneux (photo 5). Les graines des espèces plus communes (Acacia tortilis, Ziziphus mauritania, Balanites aegyptiaca) sont recueillies directement par les villageois et semées sans aucun prétraitement. Les graines des autres espèces plus rares ou difficiles à récolter (Acacia senegal, Acacia seyal, Faidherbia albida (Del.) A. Chev.) sont achetées auprès du Cnsf² à Ouagadougou. En 2007, nous avons constaté que les déjections des animaux domestiques contenaient des graines des espèces ligneuses les plus communes, telles Acacia tortilis et Ziziphus mauritania qui composent la nourriture habituelle des chèvres mais également, dans une moindre mesure, des vaches. À compter de cette date, les semis de base réalisés avec ces deux espèces (Acacia tortilis, Ziziphus mauritania) ont été effectués en jetant directement les excréments de chèvres dans les demi-lunes (photo 6).

Après quelques jours ou quelques semaines, grâce à l'eau de pluie accumulée, les graines semées mais aussi les graines des herbacées et des espèces ligneuses présentes naturellement commencent à germer, colonisant progressivement le terrain. Les terrains labourés et semés ne sont pas protégés par des clôtures. Le pâturage des animaux y est autorisé en permanence, voire encouragé, pour que les villageois puissent profiter de ce fourrage supplémentaire. Le pâturage permet en outre d'éviter une accumulation de biomasse combustible qui favoriserait les risques d'incendie en saison sèche (photo 7). surfaces à labourer (en particulier la présence de pierres en surface) et de la longueur des champs (réduction des manœuvres à vide du tracteur lorsqu'il est possible de travailler sur la longueur). D'après l'expérience acquise pendant les six dernières années dans les conditions de l'Oudalan, la capacité de travail effective du tracteur a été estimée entre 1 et 1,6 ha à l'heure, soit en moyenne 1,3 ha/heure.

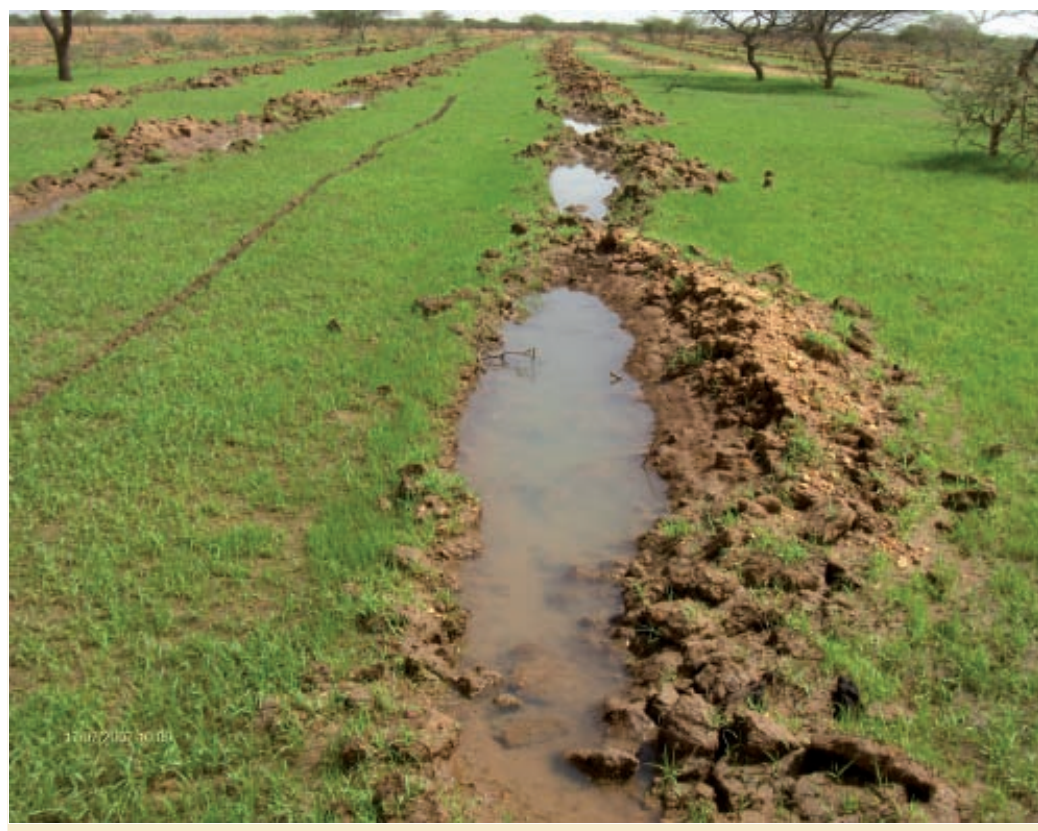

Photo 3.

Demi-lune au mois de juillet (champ I, 2007).

Photo A. Boureima. 


\section{Densité et développement aérien des arbres}

Le nombre de plantules par hectare et leur développement aérien ont été mesurés entre les années 2004 et 2009 dans cinq champs semés en 2003 (champs A-E, voir figure 2) au moyen de la canne semeuse avec des graines achetées auprès du Cnsf. Chaque année, trois placettes (échantillons non permanents de 10 lignes par 10 demi-lunes pour un total de 100 demi-lunes) choisies au hasard dans chacun des champs ont été inventoriées, soit un total de 15 échantillons par an. L'inventaire a consisté à recenser systématiquement les arbres présents dans chaque demi-lune en indiquant l'espèce et le développement de la partie aérienne mesurée en classes de hauteur de $5 \mathrm{~cm}$ (photo 8). En 2009, pour servir de témoins, des placettes de $50 \mathrm{~m}$ par $50 \mathrm{~m}$ ont été choisies au hasard dans des parcelles non labourées, voisines des champs A-E ; tous les arbres s’y trouvant ont été inventoriés.

\section{Évaluation de la biomasse herbacée}

En octobre 2003, 2005 et 2008, 50 placettes d'un mètre carré ont été placées au hasard dans chacun des champs A-E puis intégralement fauchées. Le même relevé, mais de façon ponctuelle, a été effectué dans des parcelles non labourées adjacentes. Les principales espèces herbacées ont été ensuite inventoriées et la biomasse récoltée a été séchée à l'air (12-15\% d'humidité environ) et pesée dans le but de déterminer la quantité de matière sèche en kilogrammes de matière sèche par hectare (kg MS/ha).

\section{Expérience de germination}

En 2007, le taux de germination des deux méthodes de semis, en l'occurrence les graines semées à la canne semeuse (photo 5) et l'utilisation d'excréments de chèvres dans lesquels on ignore et le nombre de graines et les espèces, a été étudié. Pour respecter les conditions naturelles, l'expérimentation a été menée in situ en champs non clôturés.

La méthode de semis des graines a été testée en utilisant des graines d'Acacia tortilis et de Ziziphus mauritania provenant chacune de deux origines différentes (récolte locale directe et achat en pépinière) pour un total de quatre traitements expérimentaux. L'expérience a été conçue en trois blocs distribués au milieu des champs I, J et K (figure 2) pour éviter des effets de lisière. Dans chacun des blocs, les quatre traitements (Acacia tortilis-pépinière, Acacia tortilis local, Ziziphus mauritania-pépinière et Ziziphus mauritania local) ont été distribués au hasard sur des placettes de 100 demi-lunes chacune (10 lignes par 10 demi-lunes). Trois semis de deux

graines ont été réalisés en trois points précis dans chaque demi-lune (aux deux extrémités et au centre), soit six graines par demi-lune ou 600 par placette.

La méthode de semis avec les excréments de chèvres a été testée dans le champ L (figure 2), dans lequel les excréments ont été éparpillés par poignées dans les demi-lunes (photo 6). En 2008, soit après un an, les mesures ont été effectuées sur trois échantillons (10 lignes par 10 demi-lunes) non permanents placés au hasard dans le champ. Le nombre de plantules présentes a été relevé ainsi que leur développement aérien dans chacune des placettes expérimentales. 


\section{Résultats}

\section{Production de biomasse dans les champs labourés}

Les données (figure 4) montrent l'hétérogénéité spatiale et temporelle du développement de la biomasse herbacée, dont le poids de matière sèche varie de 190 à $2090 \mathrm{~kg}$ MS/ha. Les variations entre les champs sont vraisemblablement dues à des différences dans la fertilité et la sensibilité à l'érosion du sol, des différences locales de pluviométrie, ainsi que dans la disponibilité en graines. Un effet temporel (temps écoulé depuis les labours, pluviométrie générale de l'année) est également visible. Les mesures ponctuelles de la biomasse herbacée sur des surfaces témoins dégradées ont donné des résultats de l'ordre de 70 à $110 \mathrm{~kg} \mathrm{MS/ha,} \mathrm{ce} \mathrm{qui} \mathrm{signifie} \mathrm{une} \mathrm{amélioration}$ de l'ordre de 3 à 30 fois pour les surfaces labourées, effet d'ailleurs visible à l'oeil nu sur le terrain (photo 9).

Les écosystèmes évoluent également du point de vue qualitatif ; au départ, la strate herbacée est dominée par Schoenefeldia gracilis, Cassia tora, Borreria stachydea, Tribulus terrestris, Mollugo nudicalis, puis apparaît un mélange plus riche d'espèces plus exigeantes et de meilleure qualité fourragère telles que Alysicarpus ovalifolius, Borreria radiata, Brachiaria lata, Cenchrus biflorus, Chloris pilosa, Citrullus lanatus, Corchorus tridens, Cyperus rotundus, Cucumis melo, Dactyloctenium aegyptium, Digitaria horizontalis, Eragrostis aspera, Eragrostis tremula, Leptadenia hastata, Leptadenia hastata, Leptadenia pyrotechnica, Mollugo nudicalis, Pennisetum pedicellatum, Zornia glochidiata, Panicum laetum, Setaria pallidefusca et Eragrostis tremula, confirmant les observations précédentes de Zoubga (2002). Cette modification de la flore herbacée contribue avec le temps à l'amélioration de la texture et de la fertilité des sols.

\section{Densité et développement aérien des arbres}

Dans les champs A-E semés en 2003 avec une poignée d'un mélange de graines d'espèces différentes, la densité des arbres est, un an plus tard, d'environ 350 arbres à l'hectare, soit un par demi-lune (avec en moyenne 348 demi-lunes par hectare). Cette valeur reste constante au cours des années ou augmente légèrement suite à la germination des graines à dormance prolongée, ou grâce à une régénération induite par le pâturage. Bien que l'analyse du taux de germination par espèce ne soit pas réalisée, il est tout de même possible d'affirmer que toutes les espèces semées sont représentées parmi les arbres inventoriés. En moyenne, plus de la moitié (54\% avec des valeurs entre $26 \%$ et $86 \%$ des demi-lunes est occupée par au moins un arbre. Comme dans le cas de la biomasse herbacée, d'importantes différences existent entre les champs en fonction de la différence de fertilité ou en raison des problèmes d'érosion qui peuvent localement être très importants. En comparaison, la densité des arbres dans les terrains non labourés est nulle dans $75 \%$ des cas (surfaces témoins des champs A, B et D) et ne dépasse jamais la densité de 108 arbres à l'hectare (témoin du champ E). Chaque espèce présente son propre rythme de croissance (figure 5). Parkinsonia aculeata, l'unique espèce non locale testée, a une croissance très rapide atteignant un développement aérien de deux mètres après quatre ans (année 2007). La croissance s'est pratiquement arrêtée en 2008 où l'on observe les premières défaillances d'individus qui séchèrent à partir de la couronne, laissant supposer l'inaptitude de l'espèce à la reforestation de la zone d'étude. A contrario, Acacia seyal, Acacia tortilis et Faidherbia albida maintiennent un rythme de croissance régulier, avec certaines différences spécifiques. Ziziphus mauritania et Balanites aegyptiaca réussissent à maintenir une croissance lente et régulière, malgré l'abrutissement le bétail.

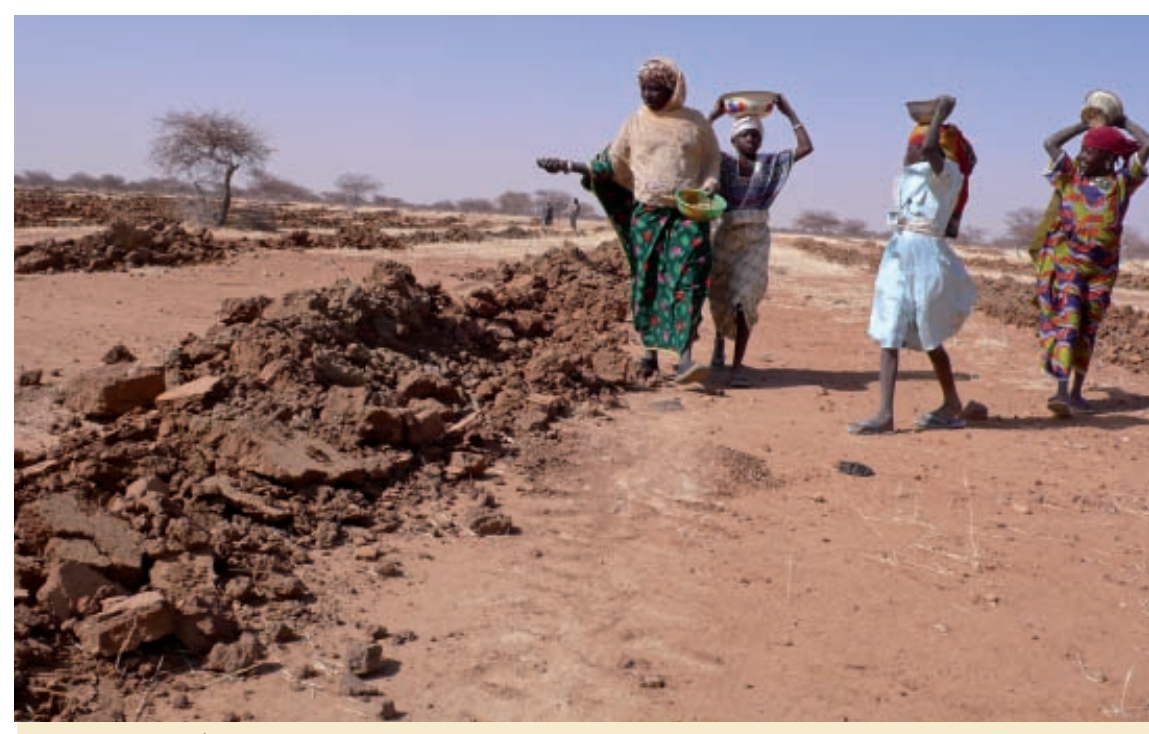

Photo 6.

Campagne de semis avec excréments de chèvres effectuée par la population locale (champ M, 2008).

Photo Deserto Verde Burkinabé.

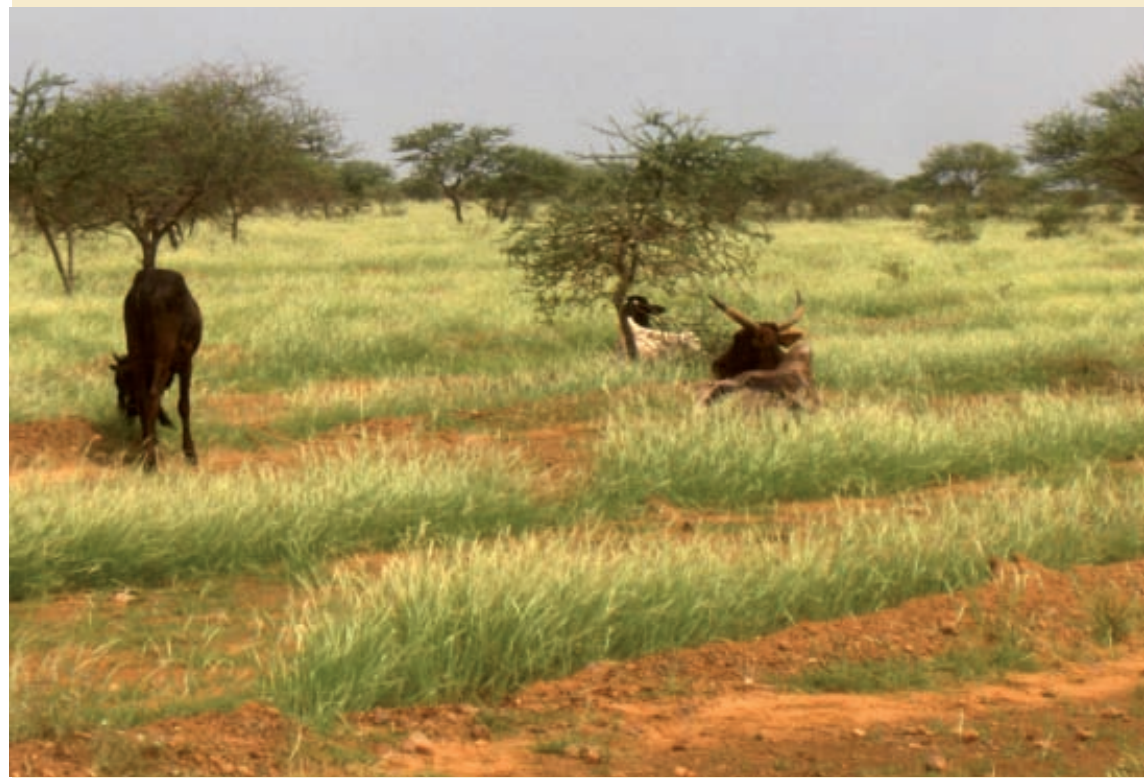

Photo 7.

Pâturage dans des champs qui ont été labourés et semés (champ A, 2004).

Photo Deserto Verde Burkinabé. 


\section{Germination des graines dans l'essai de 2007}

Les résultats du test de germination du champ K doivent être interprétés avec précaution, car celui-ci a été perturbé par un passage régulier de bétail qui a provoqué un envahissement de la placette d'Acacia tortilis par une régénération supplémentaire de la même espèce (tableau I). Dans les autres placettes, le taux de germination après un an est de 1 à $6 \%$ pour Ziziphus mauritania et de 3,5 à 6,7 \% pour Acacia tortilis, et ce indépendamment de l'origine (pépinière ou locale) des graines. La régénération complémentaire due au passage du bétail varie énormément (tableau I), ce qui est, selon toute vraisemblance, le résultat d'une intensité de pâturage différente.

Le test de semis avec excréments d'animaux

Mesure du développement aérien des arbres

(champ B, 2008).

Photo Deserto Verde Burkinabé. a donné de très bons résultats pour Acacia tortilis (en moyenne plus de 140 individus par hectare après une année) et, dans une moindre mesure pour Ziziphus mauritania (31 individus par hec-

Actuellement, aucun problème phytosanitaire majeur n'a été enregistré, exception faite pour Parkinsonia aculeata déjà cité et pour quelques individus isolés d'Acacia tortilis dans le champ C qui ont séché au cours de l'année 2007 sans qu'en soit déterminée la cause. tare) (tableau II). Le développement aérien mesuré après une saison végétative confirme les observations faites dans les champs labourés depuis 2003 (voir figure 4), avec une hauteur moyenne de $9 \mathrm{~cm}$ pour Ziziphus mauritania et de $21 \mathrm{~cm}$ pour Acacia tortilis.

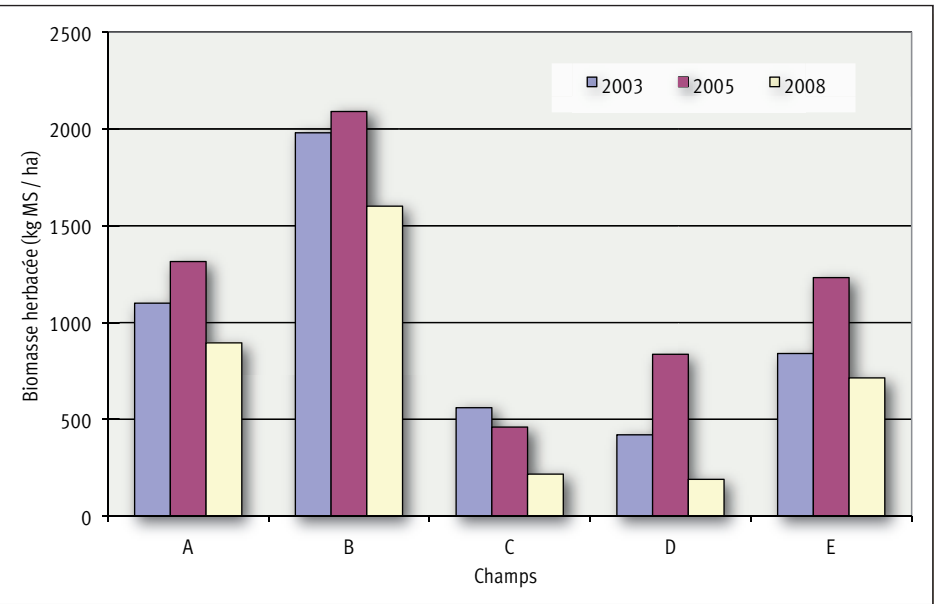

Figure 4.

Production de biomasse herbacée (en kilogrammes de matière sèche par hectare) dans les champs A-E en 2003 , 2005 et 2008

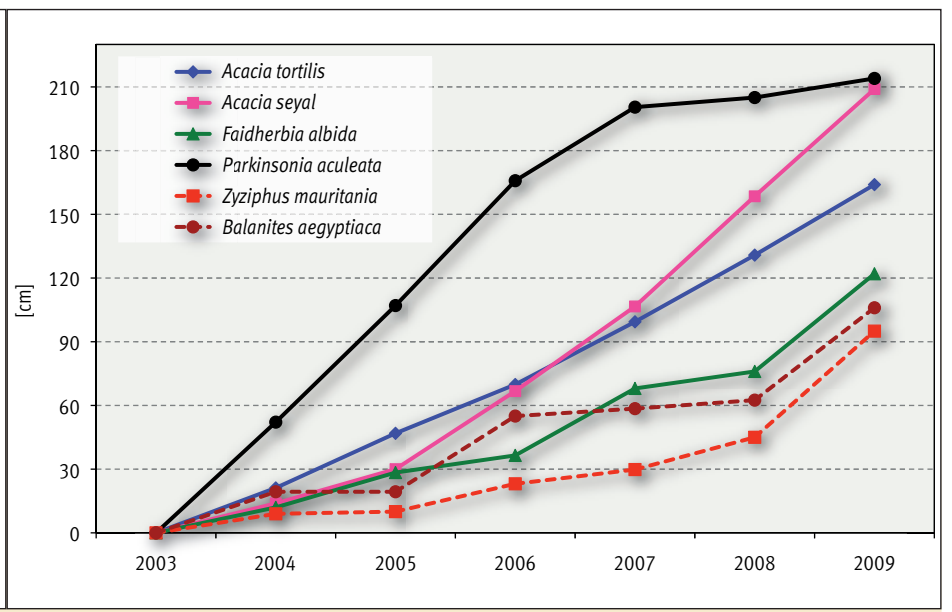

Figure 5.

Croissance des essences forestières de 2004 à 2009 (échantillons de 960 individus pour Acacia tortilis, de 70 pour Acacia seyal, de 78 pour Faidherbia albida, de 31 pour Balanites aegyptiaca, de 56 pour Ziziphus mauritania et de 25 pour Parkinsonia aculeata). Champs A-E. 


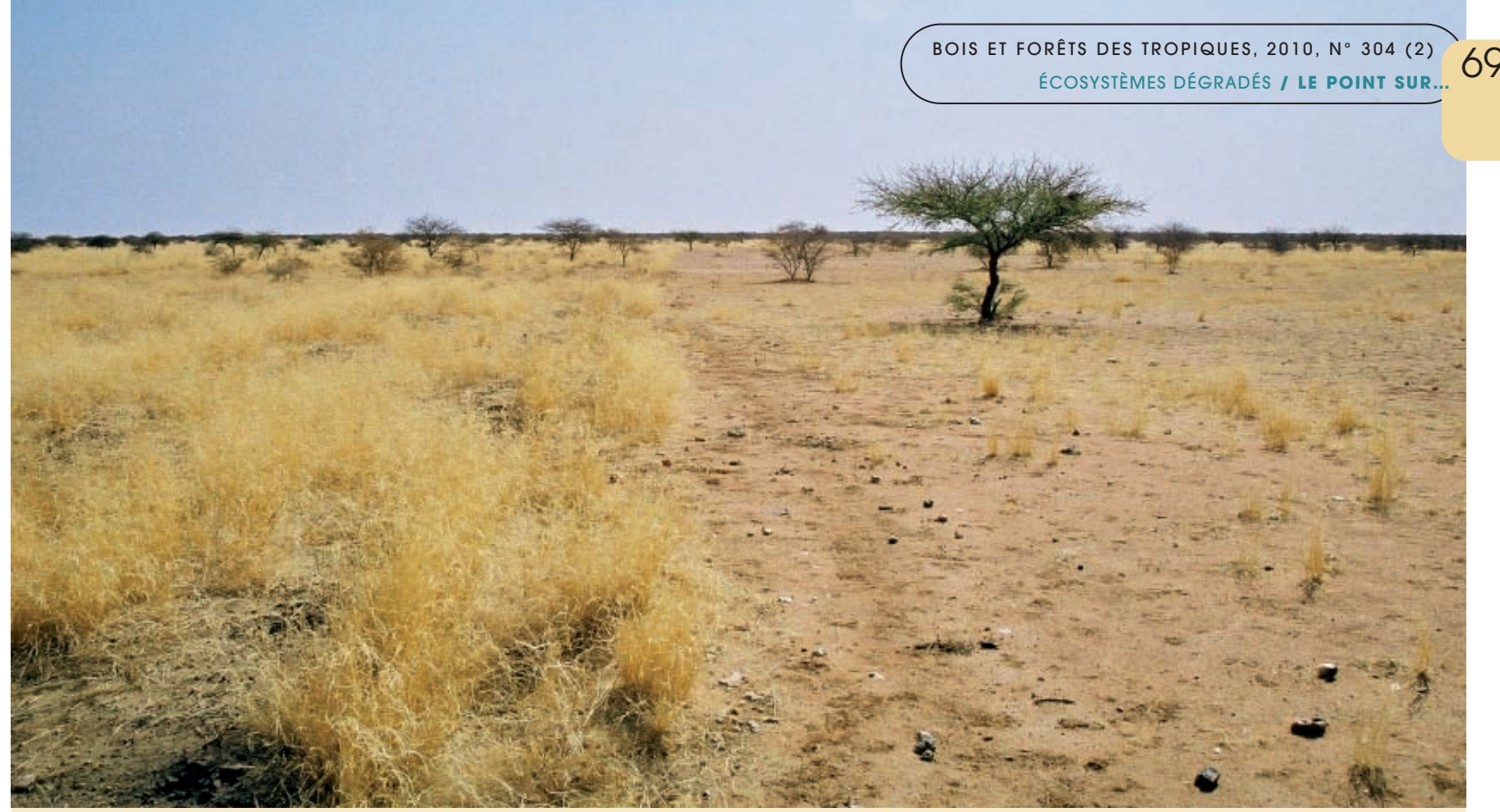

Photo 9.

Limite entre une surface labourée et surface témoin dégradée (champ B, 2004).

Photo Deserto Verde Burkinabé.

Tableau I.

Test de germination 2007 : nombre de graines ayant germé après les semis au moyen d'une canne semeuse dans les champs I, J, K (échantillons de 100 demi-lunes).

\begin{tabular}{|c|c|c|c|c|c|c|c|}
\hline & Origine & $\begin{array}{c}\text { Mode } \\
\text { de semis }\end{array}$ & I & J & K & Moyenne & $\begin{array}{l}\text { Déviation } \\
\text { standard }\end{array}$ \\
\hline Ziziphus mauritania & Pépinière & Tube & 10 & 14 & 6 & 10,0 & 4,0 \\
\hline Ziziphus mauritania & Locale & Tube & 14 & 10 & 36 & 20,0 & 14,0 \\
\hline Acacia tortilis & Pépinière & Tube & 25 & 30 & 40 & 31,7 & 7,6 \\
\hline Acacia tortilis ${ }^{1}$ & Locale & Tube & 31 & 21 & 149 & 67,0 & 71,2 \\
\hline 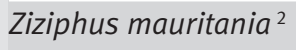 & Locale & Naturel & 0,0 & 0,0 & 1,0 & 0,3 & 0,4 \\
\hline
\end{tabular}

${ }^{1}$ Dans le champ K, la distinction entre individus semés et présents de manière spontanée est incertaine et se base uniquement sur la position de l'arbre dans la demi-lune.

${ }^{2}$ Les données représentent la moyenne du nombre d'arbres présents de manière spontanée recensés dans les quatre traitements des champs correspondants.

Tableau II.

Test de germination 2007 : nombre de graines ayant germé après épandage d'excréments d'animaux dans le champ L (échantillons de 100 demi-lunes).

\begin{tabular}{|c|c|c|c|c|c|c|c|c|}
\hline & Origine & $\begin{array}{c}\text { Mode } \\
\text { de semis }\end{array}$ & Échantillon 1 & Échantillon 2 & Échantillon 3 & Moyenne & $\begin{array}{l}\text { Déviation } \\
\text { standard }\end{array}$ & n/ha \\
\hline Acacia tortilis & Locale & Excréments d'animaux & 358 & 454 & 402 & 404,7 & 48,1 & 1408 \\
\hline Ziziphus mauritania & Locale & Excréments d'animaux & 7 & 8 & 12 & 9,0 & 2,6 & 31 \\
\hline
\end{tabular}

$\mathrm{n} /$ ha : nombre moyen de plantes à l’hectare correspondant. 


\section{Conclusion}

Nos observations confirment l'aptitude de la méthode de récupération d'écosystèmes dégradés basée sur l'emploi de la charrue Delfino dans les conditions de milieu de la zone étudiée, même lorsque les terrains sont fortement dégradés. Grâce à l'action initiale des labours, les écosystèmes sont ensuite en mesure d'inverser le processus de dégradation et de désertification. En particulier, il faut noter une augmentation significative des rendements moyens en biomasse herbacée et une amélioration de la diversité floristique, avec une disponibilité fourragère accrue, mais aussi de meilleure qualité.

Même si localement les problèmes d'érosion peuvent compromettre le processus de restauration (photo 10), en règle générale la méthode permet d'assurer la constitution d'une couverture végétale et forestière satisfaisante et proche de celle qui caractérise les écosystèmes naturels de la région. La capacité germinative des graines est conservée voire améliorée suite au passage dans l'estomac des animaux (DEPOMmIER, 1996) et les quantités d'excréments introduites constituent des apports nets de fumier organique pour ces sols pauvres en éléments nutritifs et en matière organique. La combinaison entre semis directs de déjections animales contenant des graines locales d'espèces de base (Acacia tortilis en particulier), donc plus aptes à résister à des conditions climatiques locales capricieuses, et intégration de graines d'espèces rares ou manquantes (Acacia senegal, Acacia seyal, Faidherbia albida) provenant de pépinières permet en outre d'optimiser le mélange des espèces. Malgré des rythmes de croissance et une sensibilité à l'abrutissement différents, les essences forestières semées sont toutes en mesure de croître de façon régulière, même en cas d'années de sécheresse extrême comme en 2004.
Les espèces semées forment très vite une racine pivotante qui pénètre profondément dans le terrain, assurant ainsi leur approvisionnement hydrique à long terme, lequel ne dépendra plus exclusivement de l'eau recueillie dans les demi-lunes. En effet, la structure et la capacité de rétention d'eau des demi-lunes se dégradent progressivement avec le temps, même si celles-ci restent encore visibles une décennie au moins après les labours.

La restauration d'une couverture herbacée et forestière génère d'importants effets induits sur tout l'écosystème, tels que l'augmentation des ressources alimentaires et fourragères, l'amélioration des conditions hydriques et structurelles du sol, la protection contre l'érosion éolienne et hydrique et la création d'habitats pour la petite faune (LEENDERS et al., 2007). La participation active de la population locale constitue un facteur clé pour le succès à long terme de la récupération des terres dégradées. Durant les six années d'activité de Deserto Verde Burkinabé, nous avons noté un intérêt accru des populations pour la récupération des terres dégradées dans le respect des traditions locales, comme le pâturage libre. La possibilité de pâturer les surfaces labourées dès le départ accroît l'intérêt des villageois pour cette approche.

En conclusion, il est possible d'affirmer que la dégradation des écosystèmes sahéliens peut être inversée ou ralentie à condition d'intervenir sur les facteurs limitants, tels que la réserve en eau et les conditions du lit de semis à disposition des graines au moment de la germination.

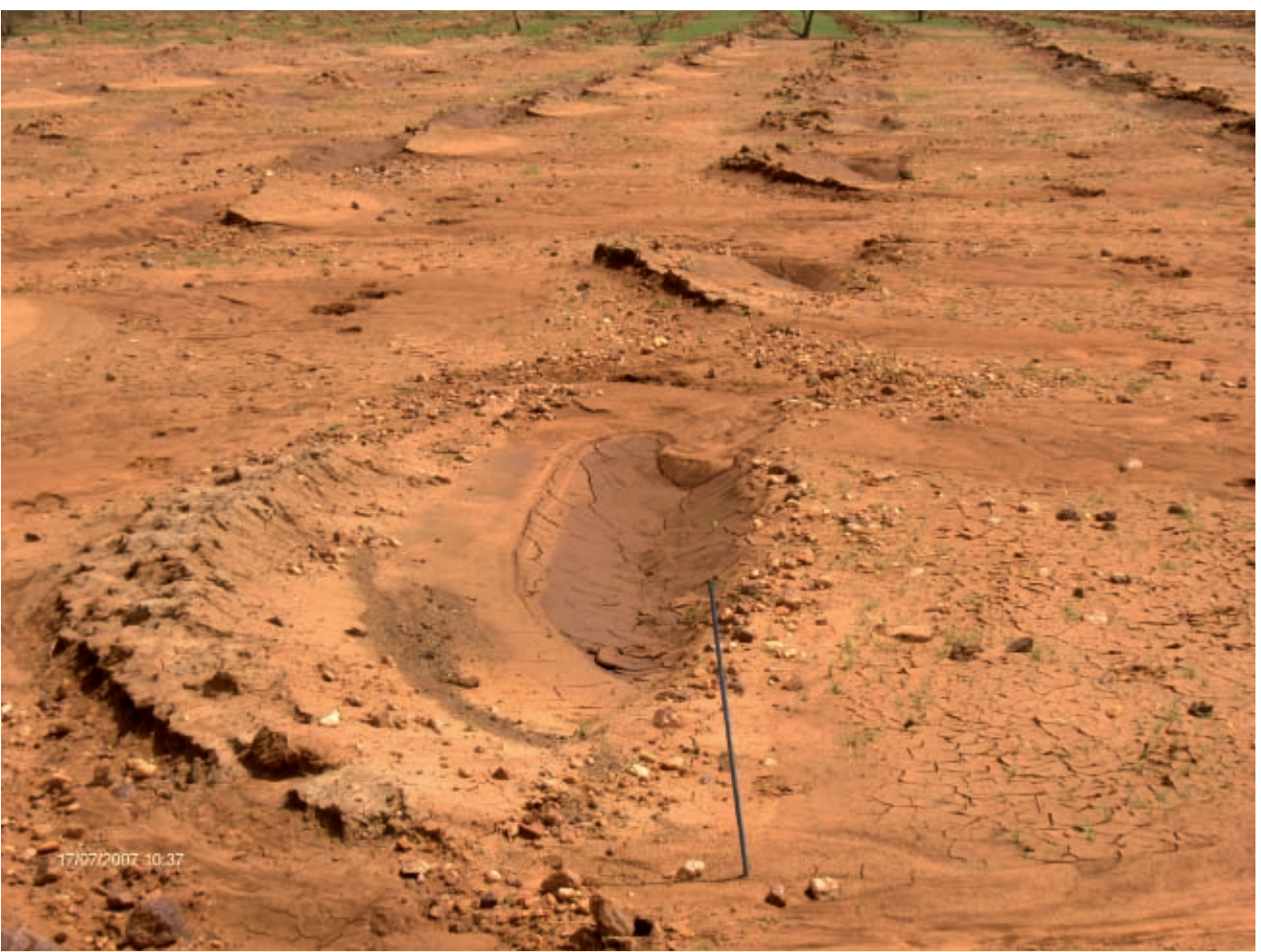

Photo 10.

Phénomènes d'érosion touchant les demi-lunes suite à la pluie (champ J, 2008). Photo Deserto Verde Burkinabé. 


\section{Remerciements}

Les auteurs tiennent à remercier les collègues bénévoles de Deserto Verde Burkinabé qui ont participé aux différentes expéditions entre 2003 et 2009, l'ingénieur Venanzio Vallerani, les collègues Jean-Pierre Sorg et Franz Schmithüsen pour la supervision critique du manuscrit, ainsi que les dirigeants et collaborateurs de Reach Italia Onlus et les villageois de Salmossi, Konsi, Gagara, Beiga, Ounare, Siloube, Wuludewu, Zougway et Gorom-Gorom.

\section{Références bibliographiques}

BOLWING S., PAARUP-LAURSEN B., 1999. Nature, work, culture : labor utilization in agriculture and off-farm employment among the Fulani in northern Burkina Faso. Danish Journal of Geography, Special Issue, 2 : 27-41.

DEPOMMIER D., 1996. Structure, dynamique et fonctionnement des parcs à Faidherbia albida (Del.) A. Chev. Caractérisation et incidence des facteurs biophysiques et anthropiques sur l'aménagement et le devenir des parcs de Dossi et de Watinoma, Burkina Faso. Thèse de doctorat, Université Pierre et Marie Curie de Paris. Montpellier, France, Cirad, 662 p.

GONZALEZ P., 2001. Desertification and a shift of forest species in the West African Sahel. Climate Research, 17 : 217-228.

GOUDET J.-P., 1985. Équilibre du milieu naturel en Afrique tropicale sèche. Végétation ligneuse et désertification. Bois et Forêts des Tropiques, 207 : 3-16.

HENRY S., SCHOUMAKER B., BEAUCHEMIN C., 2004. The impact of rainfall on the first out-migration : a multi-level event-history analysis in Burkina Faso. Population and Environment, $25: 423-459$.

HERRMANN S. M., ANYAMB A. A., TUCKER C. J., 2005. Recent trends in vegetation dynamics in the african Sahel and their relationship to climate. Global Environmental Change, 15 : 394-404.

HOUNTONDJI Y. C., SOKPON N., OZER P., 2006. Analysis of the vegetation trends using low resolution remote sensing data in Burkina Faso (1982-1999) for the monitoring of desertification. International Journal of Remote Sensing, 27 (56) : 871-884.

HULME M., DOHERTY R. M., NGARA T., NEW M. G., LISTER D., 2000. African climate change : IPCC Scientific Assessment. Cambridge, Royaume-Uni, Cambridge University Press, 364 p. IEMVT, 1977. Pâturages de l'ORD du Sahel et de la zone de délestage au nord-est de Fada N'Gourma. Maisons-Alfort, France, lemvt, 3 volumes.
LEENDERS J. K., VAN BOXEL J. H., STERK G., 2007. The effect of single vegetation elements on wind speed and sediment transport in the Saheliane zone of Burkina Faso. Earth Surface Processes and Landforms, 32 : 1454-1474.

LIKKE A. M., KRISTENSEN M. K., GANABA S., 2004. Valutation of local use and dynamics of 56 woody species in the Sahel. Biodiversity and Conservation, 1 (3) : 1961-1990.

NICHOLSON S., 2000. Land surface processes and Sahel climate. Reviews of Geophysics, 38 (1) : 117-139.

OLSSON L., EKLUNDH L., ARDÖ J., 2005. A recent greening of the Sahel-trends, patterns and potential causes. Journal of Arid Environments, 63 (3) : 556-566.

SANKARAN M., HANAN N. P., SCHOLES R. J., RATNAM J., AUGUSTINE D. J., CADE B. S., GIGNOUX J., HIGGINS S. I., LE ROUXX., LUDWIG F., ARDO J., BANYIKWA F., BRONN A., BUCINI G., CAYLOR K. K., COUGHENOUR M. B., DIOUF A., EKAYA W., FERAL C. J., FEBRUARY E. C., FROST P. G. H., HIERNAUX P., HRABAR H., METZGER K. L., PRINS H. H. T., RINGROSE S., SEA W., TEWS J., WORDEN J., ZAMBATIS N., 2005. Determinants of woody cover in African savannas. Nature, 438 : 846-849.

TOUTAIN B., GUERVILLY T., LE MASSON A., ROBERGE G., 2006. Leçons de quelques essais de régénération des parcours en région sahélienne. Sécheresse, 17 (1) : 72-75.

VALLERANI V., 2009. Un « delfino » rinverdisce il desterto. Milan, Italie, Edizioni Scripta Manent, 103 p.

WITTIG R., HAHN-HADJALI K., KROHMER J., MÜLLER J., SIEGLSETTER R., 2002. La végétation actuelle des savanes du Burkina Faso et du Bénin - Sa signification pour l'homme et la modification de celle-ci par l'homme (aperçu des résultats d'un projet de recherche). Études sur la flore et la végétation du Burkina Faso et des pays avoisinants, 7 : 3-16.

ZOUBGA T. S., 2002. Étude de l'impact du travail des charrues Delphino et Treno sur les sols et la végétation des terres dégradées du Soum (Burkina Faso). Mémoire de fin d'études, École nationale des eaux et forêts, Bobo-Dioulasso, Burkina Faso. 